\section{Severe left ventricular outflow tract obstruction with mitral regurgitation caused by accessory mitral valve tissue in an adult: A case report 1}

\author{
Sejla Sehovic (D), Djani Behram (D), Merjema Karavdic (D), \\ Sanko Pandur (D), Mirsad Kacila \\ Department of Cardiosurgery, Specialized Hospital, Heart Center; \\ Sarajevo-Bosnia and Herzegovina
}

\section{Introduction}

Accessory mitral valve tissue (AMVT) is an extremely rare congenital cardiac abnormality in adults. Patients typically present with symptoms and signs of left ventricular (LV) outflow tract (LVOT) obstruction. LVOT obstruction caused by AMTV was diagnosed mostly in the first decade of life. However, isolated cases of AMVT with LVOT obstruction in adulthood are uncommon $(1,2)$. This paper reports a case of an adult patient aged 56 years with severe LVOT obstruction and severe mitral regurgitation (MR) caused by AMVT.

\section{Case Report}

A 56-year-old man with progressive exertional dyspnea was referred to our cardiosurgery center for evaluation of LVOT obstruction and MR. Clinical examination on admission revealed a grade IV systolic heart murmur in the $5^{\text {th }}$ intercostal space that propagated to the left axillary region. His blood pressure on admission was $128 / 79 \mathrm{~mm} \mathrm{Hg}$, pulse rate was 62 beats per minute, and his lungs were clear on auscultation. A chest X-ray showed a slightly enlarged cardiac silhouette, and both lungs were clear with no obvious infiltrates or pleural effusions. An electrocardiogram showed sinus rhythm with signs of LV hypertrophy. Our patient complained of progressive exertional dyspnea for the last five months.

Transthoracic echocardiography (Fig. 1 and Video 1) in our center showed marked concentric LV hypertrophy, normal LV systolic function, and an abnormal tissue on the mitral valve extending to the LVOT, raising the pressure gradient (PG) flow through the LVOT (LVOT maximum PG of $85 \mathrm{~mm} \mathrm{Hg}$ ).

To obtain more detailed information, transesophageal echocardiography (TEE) was performed (Fig. 2 and Video 2). TEE revealed a morphologically altered mitral valve with elongated chords of the anterior mitral leaflet, that is, AMVT. AMVT was documented as an abnormal string-like structure extending from the annular region, attached to the tip of the anterior mitral valve leaflet, and protruding into the LVOT during systole, causing LVOT obstruction. Color flow revealed a mosaic flow pattern, suggesting a mechanical obstruction in the LVOT. The maximum PG across the LVOT (LVOT maximum PG of $85 \mathrm{~mm} \mathrm{Hg}$ ) was documented as well as the severe type of MR with a wide eccentric regurgitation jet that filled the entire atrium (continuous wave Doppler pattern wide, MR maximum $P G$ of $110 \mathrm{~mm} \mathrm{Hg}$, and vena contracta width of $0.75 \mathrm{~cm}$ ).

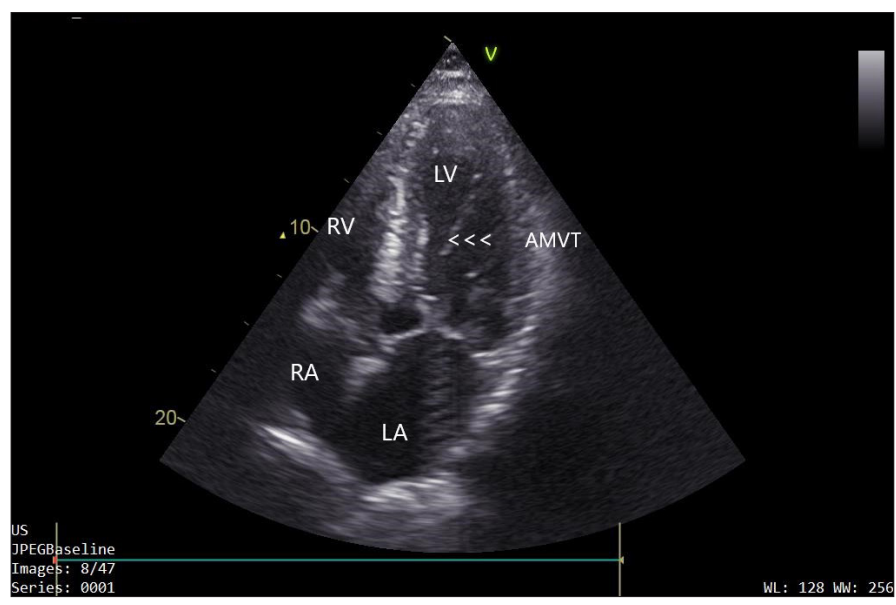

Figure 1. TTE (apical four-chamber view) showing abnormal string-like tissue, that is, AMVT (white arrow)

AMVT - accessory mitral valve tissue; TTE - transthoracic echocardiogram

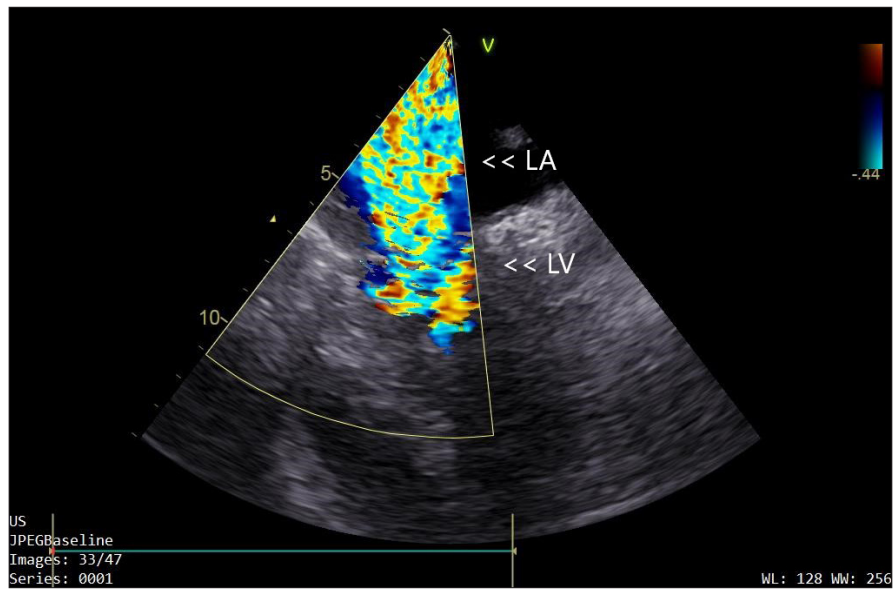

Figure 2. Severe mitral regurgitation-wide eccentric regurgitant jet extended into the entire LA

LA - left atrium

Surgical treatment was recommended on the basis of echocardiographic evidence of significant LVOT obstruction secondary to AMVT and severe MR.

Intraoperative findings showed preserved myocardial contractibility with an enlarged left heart and a hypertrophied left ventricle. $O n$ the ventricular sides of the anterior mitral valve leaflet in the area of $A 2$ and $A 3$, a structure previously described as AMVT was attached. The AMVT was surgically removed, and the mitral valve was replaced (with mechanical mitral valve No 31), with complete resolution of LV outflow tract gradient and symptoms.

\section{Discussion}

AMVT is a very rare cardiac abnormality in adults. LVOT obstruction caused by AMTV was diagnosed mostly in the first decade of life. However, isolated cases of AMVT with LVOT obstruction in adulthood are uncommon with an estimated incidence of 1 per 26,000 echocardiograms (1-3). According to research data, 
the oldest patient was diagnosed with AMVT at the age of 77 years (4). Our case is an adult symptomatic patient aged 56 years with severe LVOT obstruction and severe MR caused by AMVT.

Two possible mechanisms underlying LVOT obstruction in patients with AMVT were reported (5): systolic ballooning of the AMVT into the LV outflow tract leads to the large effect of the AMVT and progressive deposition of fibrous tissue owing to the perturbing flow caused by AMVT $(4,5)$.

Patients with AMVT may be asymptomatic with the presence of a murmur, or more often, they experience symptoms $(5,6)$. Usually, patients become symptomatic when the mean gradient across the LVOT reaches $50 \mathrm{~mm} \mathrm{Hg}(4,5)$. Signs of LVOT obstruction generally develop during the first decade of life in one third of the patients. However, our patient, presented in this report, did not develop symptoms of LVOT obstruction until age 56 years.

In our patient, the possible reasons why the PG in the LVOT was not present until his 50 s could be additionally explained with the compensatory mechanism (4). The AMV did not prolapse into the LVOT during childhood, but the chordae gradually became longer, and finally, the valve tissue prolapsed into the LVOT, which resulted in a prominent systolic heart murmur (4). In our case, we observed elongated chordae tendinae on TEE.

It is hypothesized in the literature that chordae tendineae elongation occurs as part of the compensatory remodeling, which suggests that the chords remodel as a compensatory mechanism for preventing MR to increase $(5,7)$.

We emphasize in our case that TEE presents a cornerstone diagnostic tool for the diagnostic and follow-up of patients with this rare malformation.

Although both transthoracic echocardiogram (TTE) and TEE are the gold standards in the diagnosis and evaluation of AMVT. TEE is a critical tool to provide more detailed information of the LVOT and to define better morphology of the mitral valve and the attachment points of the AMVT, especially in patients scheduled for surgery (Fig. 2 and Video 2).

Moreover, as highlighted in our case report, TEE allows the identification of AMVT not seen on TTE examination. The presence of significant LVOT obstruction should always lead to a surgical referral even in asymptomatic cases before complications, such as thromboembolism, LV hypertrophy, and eventually LV dilatation with impaired systolic function, occur (8-10). Variable degree of MR may be often present (8). In our patient, a mitral valve replacement was conducted because the AMVT could not be accessed until after the excision of the anterior mitral valve leaflet. Postoperative echocardiography showed complete relief of the mitral valve leaflets with a wide resolution of LV outflow tract gradient and accompanied MR.

\section{Conclusion}

Our case highlights the pivotal role of TEE in the diagnosis of this cardiac anomaly and in the preoperative planning of successful surgical treatment.

In summary, AMVT should be suggested in patients with LVOT obstruction. Despite being a rare cardiac malformation,
AMVT should always be considered in the differential diagnosis of LVOT obstruction. AMVT may cause LV outflow tract obstruction mimicking different other causes of obstruction.

Informed consent: Written informed consent was obtained from the patient.

Video 1. TTE (apical four-chamber view) showing abnormal stringlike tissue, that is, AMVT (white arrow)

AMVT - accessory mitral valve tissue; TTE - transthoracic echocardiogram

Video 2. TEE finding (mid-esophageal view) showing the accessory mitral valve tissue - a highly mobile string-like structure (the arrowhead) —on the ventricular side of the anterior mitral valve leaflet prolapsing into the LVOT. Note the concentric hypertrophy of the left ventricle

LVOT - left ventricular outflow tract; TEE - transesophageal echocardiography

\section{References}

1. Rovner A, Thanigaraj S, Perez JE. Accessory mitral valve in an adult population: the role of echocardiography in diagnosis and management. J Am Soc Echocardiogr 2005; 18: 494-8. [Crossref]

2. Prifti $\mathrm{E}$, Bonacchi M, Frati G, Voci P, Leacche M. Accessory mitral valve leaflet in an adult with coronary artery disease. J Cardiovasc Surg (Torino) 2002; 43: 843-7.

3. Yuan SM, Shinfeld A, Mishaly D, Haizler R, Ghosh P, Raanani E. Accessory mitral valve tissue: a case report and an updated review of literature. J Card Surg 2008; 23: 769-72. [Crossref]

4. Panduranga $\mathrm{P}, \mathrm{Al}$-Mukhaini $\mathrm{M}$. Isolated non-obstructive accessory mitral valve tissue in an adult mimicking ruptured chordae. Indian Heart J 2013; 65: 334-6. [Crossref]

5. Aoka Y, Ishizuka N, Sakomura Y, Nagashima H, Kawana M, Kawai A, et al. Accessory mitral valve tissue causing severe left ventricular outflow tract obstruction in an adult. Ann Thorac Surg 2004; 77: 713-5. [Crossref]

6. Manganaro R, Zito C, Khandheria BK, Cusmà-Piccione M, Chiara Todaro M, Oreto $\mathrm{G}$, et al. Accessory mitral valve tissue: an updated review of the literature. Eur Heart J Cardiovasc Imaging 2014; 15: 489-97. [Crossref]

7. Obase K, Weinert L, Hollatz A, Farooqui F, Roberts JD, Minhaj MM, et al. Elongation of chordae tendineae as an adaptive process to reduce mitral regurgitation in functional mitral regurgitation. Eur Heart J Cardiovasc Imaging 2016; 17: 500-9. [Crossref]

8. Tanaka H, Kawai H, Tatsumi K, Kataoka T, Onishi T, Yokoyama M, et al. Accessory mitral valve associated with aortic and mitral regurgitation and left ventricular outflow tract obstruction in an elderly patient: a case report. J Cardiol 2007; 50: 65-70.

9. Sharma R, Smith J, Elliott PM, McKenna WJ, Pellerin D. Left ventricular outflow tract obstruction caused by accessory mitral valve tissue. J Am Soc Echocardiogr 2006; 19: 354.e5-8. [Crossref]

10. Kim MS, Klein AJ, Groves BM, Quaife RA, Salcedo EE. Left ventricular outflow tract obstruction in the presence of asymmetric septal hypertrophy and accessory mitral valve tissue treated with alcohol septal ablation. Eur J Echocardiogr 2008; 9: 720-4. [Crossref]

Address for Correspondence: Sejla Sehovic, MD, Specialized Hospital, Heart Center, Department of Cardiosurgery, Kranjceviceva 12,71000, Sarajevo-Bosnia and Herzegovina

Phone: 0038761278589

E-mail: sejlas10@hotmail.com

CCopyright 2021 by Turkish Society of Cardiology -

Available online at www.anatoljcardiol.com

D0I:10.5152/AnatolJCardiol.2021.40088 